UDC 519.925.51

DOI: $10.20535 /$ SRIT.2308-8893.2020.1.08

\title{
OPTIMAL STOCK PORTFOLIO DIVERSIFICATION UNDER MARKET CONSTRAINTS
}

\author{
V. KULIAN, M. KOROBOVA, O. YUNKOVA
}

\begin{abstract}
The problem of optimal portfolio diversification is considered. Based on mathematical models of the dynamics of the market value formation of a single share and an optimal stock portfolio, the structure of the optimal portfolio is determined. Such models are built in a class of ordinary differential equations. One of the problems of optimal investing is optimizing the expected return of the stock portfolio for the desired level of risk. Another problem is the choice of the stock portfolio with the same expected return, but with a smaller risk. For this purpose, we use a set of acceptable and effective portfolios. This sequence of steps of the algorithm allows consistently solve two optimization problems. The problem of portfolio diversification consists of the problem of determining the moments of time and the necessity to perform such a diversification. In the article, we constructed an algorithm for determining these points of time, based on the solution of an optimal control problem. The application of this algorithm enables to select an optimal risk portfolio at a certain level of its expected profitability. It uses an efficient and acceptable set of investment portfolios.
\end{abstract}

Keywords: optimal portfolio, diversification, admissible set, effective set.

\section{THE GOAL OF THE WORK}

In this paper an attempt to construct new fundamental approaches for solving portfolio investment problems, based on the application of dynamic systems mathematical modeling and the admissible and effective set of portfolio is made.

Main purpose of this paper is to apply mathematical modeling methods and management theory to solve dynamic investment management problems, to explore asset and liability portfolio management strategies, and in general financial instruments in a dynamic case. We develop analytical methods and computational procedures for solving the problem of two-criterion optimization of a portfolio of risky securities. The problems are presented in the formulation of $\mathrm{H}$. Markowitz [1] in the presence of quantitative and qualitative instrumental market constraints on the structure of the portfolio. It's important to say, that an alternative approach to solving the problem of investment optimization and which has proved to be quite effective is to use the apparatus of fuzzy sets $[6,7]$.

\section{MATHEMATICAL FORMALIZATION AND CONSTRUCTION OF MODELS}

Dynamic Asset and Liability Management (ALM) [1] models have found the most successful use in long-term financial planning, where the need for multiple decision-making is determined by the essence of the process. Examples of ALM work include implemented models for pension funds, insurance companies, investment companies, banks, university funds [1]. 
The general scheme of active management of a stock portfolio, as a rule, consists of the following steps:

1. Calculation of the price of an individual share on the basis of static deterministic models.

2. Analysis of static stock portfolio.

3. Modeling of asset dynamics.

4. Modeling the dynamics of the asset portfolio.

5. Integrated management and diversification of assets portfolio.

In order to move from the problem of static to dynamic consider Sharp's market model

$$
r=\alpha+\beta S M_{\text {ind }}+\varepsilon,
$$

where $r$ - the market price of the stock; $S M_{\text {ind }}$ - stock market index; $\alpha-$ some basic value for $r$. The equation describes the only general principles for determining market value of a stock. From the above correlation it can be seen that the market value of the stock is formed by the integral influence of the stock market index and the random component. The ratio can be formally regarded as a characteristic of the impact of the market index on the formation of one share market value. Given that such processes occur over time, we can write down

$$
r(t)=\alpha(t)+\beta S M_{\text {ind }}(t)+\varepsilon .
$$

It would be a great simplification, when modeling such a complex process, to consider function as the primary and the only one that generates value. The analysis of the factors influencing the dynamics of the process gives grounds to argue that such significant factors can also be attributed to the correlation between securities and inflation. So let's write it in the form

$$
r_{i}(t+\delta t)=r_{i}(t)+\beta\left(S M_{\text {ind }}(t), I(t), \rho_{i j}, r_{i}(t)\right) \delta t, i, j=\overline{1, n} .
$$

Given the above, we write the right part in the form

$$
\begin{gathered}
r_{i}(t+\delta t)=r_{i}(t)+\left(\left(\alpha_{1} S M_{\text {ind }}(t)+\alpha_{2} I(t)\right) r_{i}(t)+\sum_{j=1}^{n} \rho_{i j} r_{i}(t)\right) \delta t, \\
\frac{r_{i}(t+\delta t)-r_{i}(t)}{\delta t}=\left(\alpha_{1} S M_{\text {ind }}(t)+\alpha_{2} I(t)\right) r_{i}(t)+\sum_{j=1}^{n} \rho_{i j} r_{j}(t), i=\overline{1, n .}
\end{gathered}
$$

Modern securities trading technologies and conditions of formation of market value of shares on the stock market make it possible to take the next step

$$
\frac{d r_{i}}{d t}=\left(\alpha_{1} S M_{\text {ind }}(t)+\alpha_{2} I(t)\right) r_{i}(t)+\sum_{j=1}^{n} \rho_{i j} r_{j}(t), \quad i=\overline{1, n},
$$

where $\alpha_{1}, \alpha_{2}$ - model parameters.

In the case $\rho_{i j}, i=\overline{1, n}$, is also a vector of model parameters that takes into account correlations between stocks. Here $n-$ the number of shares with which it correlates $i$ - share.

In the time interval $t \in\left[t_{0}, t_{1}\right]$ equation that describes the return of the stock portfolio $r_{p}$, looks like

$$
r_{p}(t)=\sum_{i} x_{i}(t) r_{i}(t)
$$


where $x_{i}$ - part of shares $i$ - kind in the portfolio; $r_{i}$ - expected return on shares of $i-$ kind. Differentiating both parts by $t$, we will get

$$
\frac{d r_{p}}{d t}=\sum_{i}\left(r_{i}(t) \frac{d x_{i}(t)}{d t}+x_{i}(t) \frac{d r_{i}(t)}{d t}\right. \text {. }
$$

Let us consider that for $i \neq j$ there are relationships

$$
\begin{gathered}
\sum_{i} x_{i}(t) r_{i}(t) \frac{f_{i}}{r_{i}(t)}=\sum_{i} x_{i}(t) r_{i}(t)-\sum_{i} \sum_{j} x_{i}(t) r_{i}(t) \frac{f_{j}}{r_{j}(t)}, \\
\sum_{i} \frac{x_{i}(t) r_{i}(t)}{x_{i}(t)} \frac{d x_{i}(t)}{d t}=\sum_{i} x_{i}(t) r_{i}(t)-\sum_{i} \sum_{j} x_{i}(t) r_{i}(t) \frac{d x_{j}(t)}{d t} \frac{1}{x_{j}(t)} .
\end{gathered}
$$

Function $f$ is the right part of the mathematical model of stock price formation [3]

$$
\frac{d r_{i}}{d t}=\left(\alpha_{1} S M_{\text {ind }}(t)+\alpha_{2} I(t)\right) r_{i}(t)+\alpha_{3}(x(t), r(t)) .
$$

Then the dynamic equation of pricing of the stock portfolio will look like

$$
\frac{d r_{p}(t)}{d t}=2 r_{p}(t)-\sum_{i} \sum_{j} x_{i}(t) r_{i}(t)\left(\frac{f_{j}}{r_{j}(t)}+\frac{d x_{j}(t)}{d t} \frac{1}{x_{j}(t)}\right), i \neq j .
$$

The last ratio, assuming the above, describes the dynamics of the behavior of a portfolio of risky securities. His more detailed analysis points to two important features that characterize the market value of the portfolio and that it depends on the dynamics of both the expected return on the shares and the change in the structure of the portfolio.

For solving and analyzing applied portfolio investment problems there are wide range of approaches [2], [3]. A significant part of them involves the active use of technical analysis methods, which make it possible to determine the market value of the stock in the future. Such rules for constructing the forecast, due to the well-developed mathematical formalizations and approaches and relatively not complicated practical implementation, are actively developing and effectively applied not only in the stock market. An application of fundamental analysis analytical methods allows for us to answer the question: why the market value of a stock in the future will be just such? At present, due to the complexity of mathematical models in the study of the market pricing processes of stock market assets, the methods of fundamental analysis have not yet found effective development and constructive application. Principles for analyzing processes based on the development and application of mathematical modeling methods [2-6] are obviously the most promising and devote much attention to the research.

\section{MATHEMATICAL FORMULATION OF THE PROBLEM}

The mathematical problem of constructing the optimal dynamics of the portfolio of shares in the most general formulation of H. Markowitz has the form [1] 


$$
\left\{\begin{array}{c}
r^{\mathrm{T}} x=\underset{x}{\max ,} \\
x^{\mathrm{T}} V x \rightarrow \min , \\
x \\
I^{\mathrm{T}} x=1, \\
x_{i} \geq 0, \quad i=\overline{1, n} .
\end{array}\right\} .
$$

Here $\mathrm{T}-$ is the sign of transposition.

The content of this two-criterion task (1) is to determine the optimal investment strategy, which involves maximizing the expected profitability and minimizing the risk at the same time. According to H. Markowitz, the criteria in the task are controversial, that is, improving the outcome of one of them leads to deterioration beyond others. In practice, this means that increasing the profitability of a portfolio corresponds to an increase in its riskiness. There are different approaches to solving the problem (1), but they are more academic in nature and difficult to apply to real investment in securities. A step that can bring the problem formulation (1) closer to the practical investment needs divide this twocriterion problem into two one-criterion ones. The first of them involves risk optimization at a predetermined level of expected profitability at the chosen time point, and the second is the optimization of the expected profitability for the investor-defined "optimal" portfolio risk level. In some cases, such mathematical statements of nonlinear programming problems allow for analytical solutions [2], but do not consider the essential features. They consist in the fact that at each step the solution of the problem of diversification of the portfolio of portfolios must be taken into account as budgetary and instrumental constraints. The constraints make possible to analyze the availability of the required quantity and quality of financial instruments on the market

$$
x_{i}(t) \in X(t), \quad i=\overline{1, n} .
$$

Here $X(t)$ - limited set of admissible portfolios. The mathematical formulation of the problem of optimizing the risk of an investment portfolio at a time point determined by the level of its expected profitability is

$$
\left\{\begin{array}{l}
r^{\mathrm{T}}(T) x(T)=r_{P}(T), \\
x^{\mathrm{T}}(T) V x(T) \rightarrow \min _{x}, \\
I^{\mathrm{T}} x(T)=1, \\
x_{i} \geq 0, i=\overline{1, n}, t \in\left[t_{0}, T\right], \\
x_{i}(t) \in X(t), i=\overline{1, n}, t \in\left[t_{0}, T\right] .
\end{array}\right\} .
$$

On an example of investing in a stock, we consider the problem of optimizing portfolio risk for a given level of its expected return (3), while taking into account the constraints (2). 


\section{THE PROBLEM OF OPTIMAL PORTFOLIO DIVERSIFICATION}

The optimal diversification procedure consists of successive steps, each of which makes the decision on the best one, taking into account the limitations of the structure of the stock portfolio.

The first step in solving the problem is to identify the times at which it is appropriate to diversify the portfolio. For this purpose, we will use Algorithm 2 [3] to solve the problem of determining the optimal moments of stock portfolio diversification. The algorithm procedure allows to determine consecutive time points that divide the study interval into separate intervals, each of them builds optimal trajectories of acceptable portfolios in the presence of market constraints on the portfolio structure. The mathematical problem of optimizing a stock portfolio based on a programme trajectory and possible algorithms for solving it are described in [3]. A graph showing an example of the interval breakdown and corresponding trajectories at each time interval is given on fig. 1 .

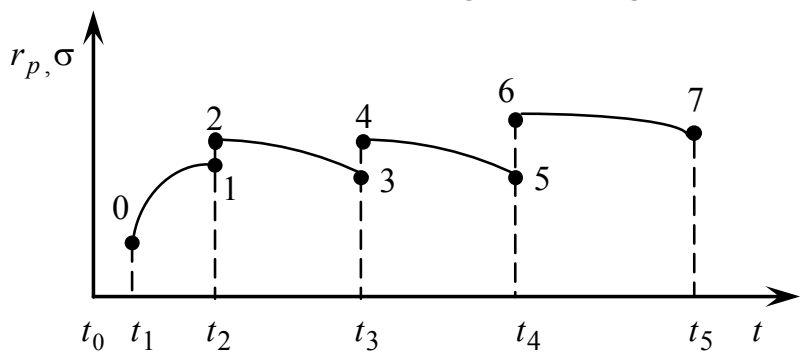

Fig. 1. Stock portfolio trajectories that are optimal over time

On the basis of modeling the dynamics of one share market value and the portfolio of shares, the task of optimal management of different types of shares in the portfolio is solving. The result of this task is trajectories $0-1,2-3,4-5,6-7$.

The next step is to build an optimal diversification procedure for the investment portfolio at each individual interval. General task of H. Markowitz of risky assets portfolio optimization (1) involves consideration of another criterion - riskiness. Let's use this in the future by the set of admissible and effective portfolios. At the same time, one of the main tasks of portfolio investment is the problem of optimal diversification of such portfolio.

The risk optimization procedure for optimal portfolio profitability is to select at each step the permissible portfolios that lie on the appropriate line. This line connects the point, that corresponds to the optimal market value of the portfolio with the point, belonging to the effective set. This line is parallel to the axis of riskiness of portfolios. The feature of this choice of optimal portfolio is that in this line, according to the definition, each of the portfolios corresponds to the same expected profitability, but the risk decreases in the direction of the axis. This property of the admissible set of investment portfolios allows from one side to take into account the limitations (2) and on the other — to determine the portfolio of "optimal" expected returns with less risk. For $t_{1}$ time moment admissible and effective sets will looks like as on a fig. 2 .

In fig. 1 points 1 and 2 are defined for the time moment $t_{1}$. Point 1 is the optimal portfolio for the moment, calculated on the basis of solving the problem of optimal portfolio management structure using one of the algorithms described 
in [3]. In order to obtain the initial optimal portfolio value for the next time moment $t_{2}$, we apply a valid and effective set.

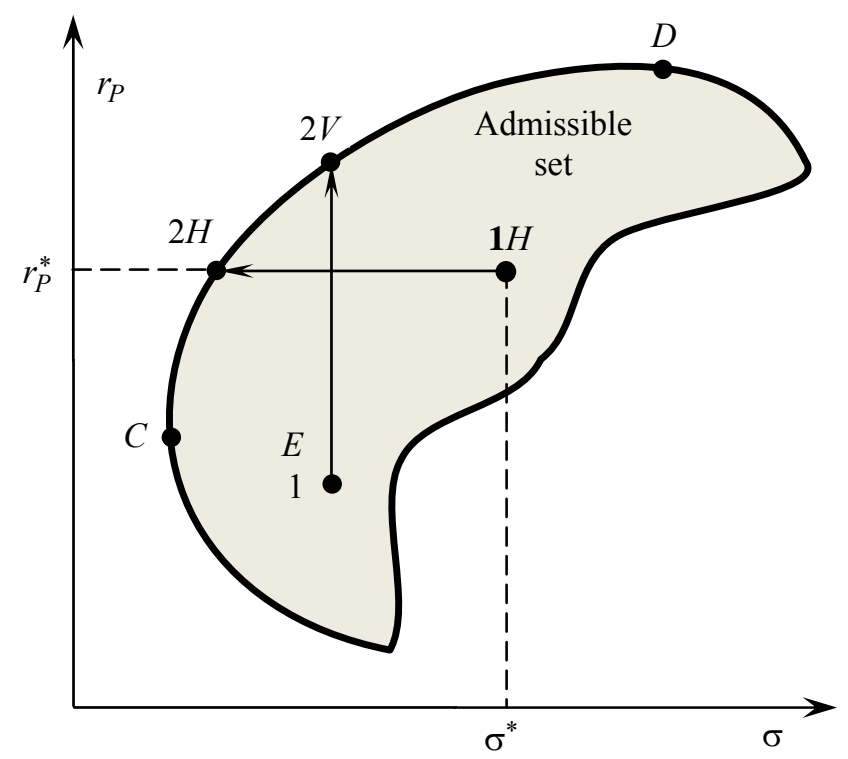

Fig. 2. Solving the problem of optimizing the stock portfolio risk for $t_{1}$ time moment

In line 1-2, fig. 2, depending on the formulation of the problem, choose the optimal value of the initial value for the next interval portfolio value. It should be noted that one of the tasks may involve determining the optimal value of the expected return for a given level of risk, and the other - on the contrary. Thus, the built-in algorithm of diversification of the optimal stock portfolio makes it possible to take into account the instrumental and quantitative constraints in the construction of the optimal stock portfolio that emerges on the market at any given time.

We will apply this procedure to build an optimal stock portfolio for the following time intervals.

For $t_{2}$ time moment admissible and effective sets will looks like as on a fig. 3.

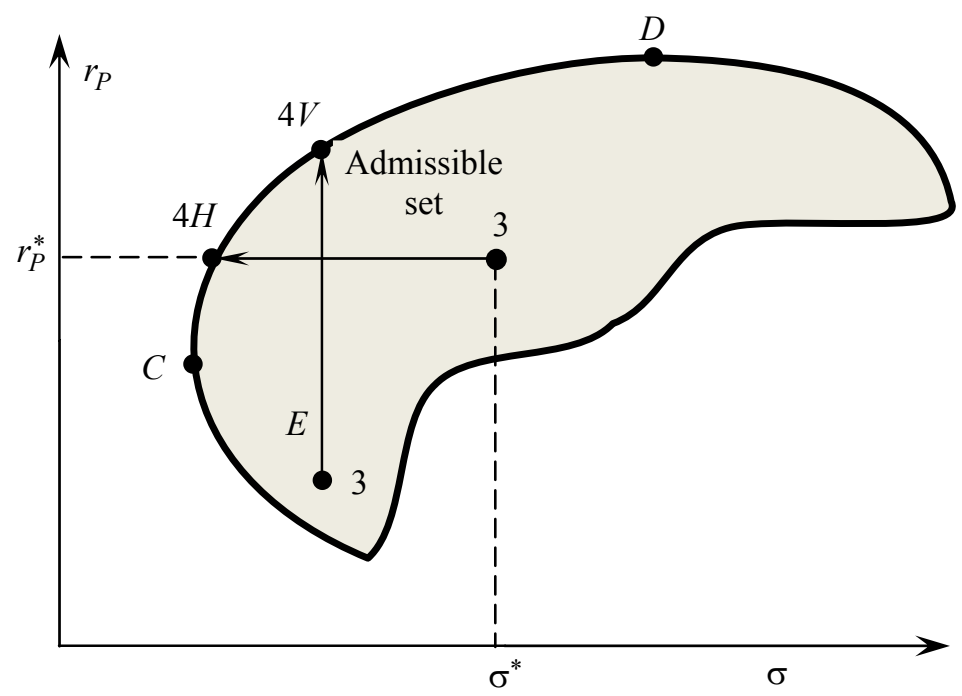

Fig. 3. Solving the problem of optimizing the stock portfolio risk for $t_{2}$ time moment 
For $t_{3}$ time moment admissible and effective sets will looks like as on a fig. 4.

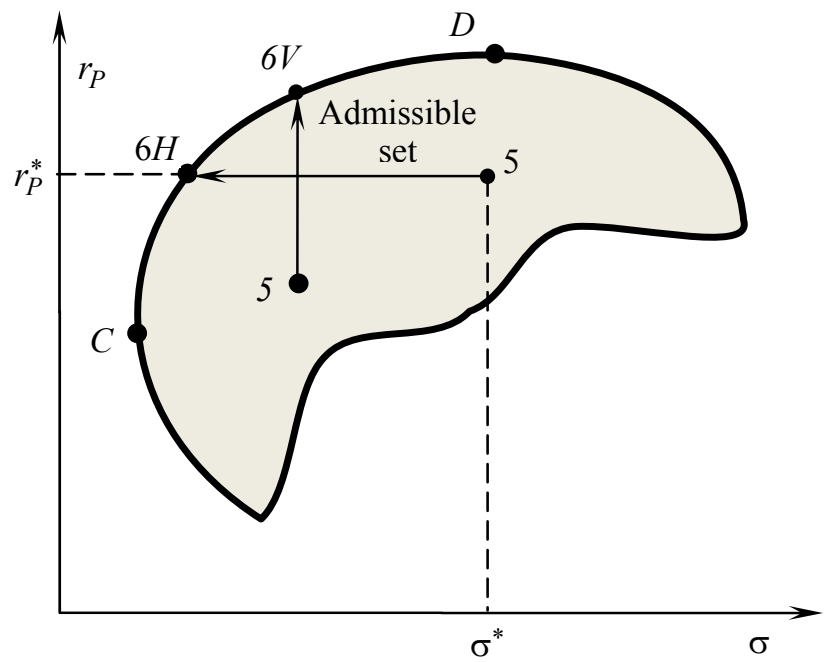

Fig. 4. Solving the problem of optimizing the stock portfolio risk for $t_{3}$ time moment

Let's turn to the second task in the general statement of $\mathrm{H}$. Markowitz about optimization of risk optimal for the expected profitability of the portfolio of shares. To do this, we use the sets of admissible and effective portfolios that correspond to the selected set of shares [1,2].

If a certain portfolio is at a point 5 , that is, for which there is no risk of reducing according to the above rule, then we define the "optimal portfolio" by moving it from point 5 to point $6 \mathrm{~V}$ that is an element of an effective portfolio of portfolios. In fact, this means the definition of a portfolio of stocks with a higher expected return. At the same time, such a procedure allows constructively to take into account the existing limitations when diversifying the portfolio.

Another mathematical statement of the problem of optimizing the expected return on the investment portfolio at a time point determined by the level of its risk, is such

$$
\left\{\begin{array}{l}
r^{T}(T) x(T) \rightarrow \max _{x}, \\
x^{T}(T) V x(T)=\tau, \\
I^{T} x(T)=1, \\
x_{i} \geq 0, i=\overline{1, n}, t \in\left[t_{0}, T\right], \\
\mathrm{x}_{\mathrm{i}}(t) \in X(t), i=\overline{1, n}, t \in\left[t_{0}, T\right] .
\end{array}\right\} .
$$

The procedure for optimizing the expected return on a portfolio for a certain level of its risk is to select a procedure for optimizing the expected return of a portfolio for a certain level of its risk is to select at each step the admissible portfolios that lie of the straight line 5-6 (fig. 4). The line connects the points 5 that corresponds to the optimal expected return on the calculated portfolio and the point 6 belonging to the effective set. This line is parallel to the axis of market value. The feature of this choice of optimal portfolio is that in this line, according 
to the definition, each of the portfolios is responsible for the same risk, but the market value increases.

This property of the admissible set of investment portfolios, as in the previous case, allows one to take into account the restrictions (2), on the other hand, to define a portfolio with "optimal" risk and higher expected returns.

As defined above, portfolio is at a point 5 , that is, for which there is no possibility to increase the expected yield, according to the above rule, then we determine the "optimal portfolio" by moving it from point 5 to point $6 H$ that is an element of an effective portfolio of portfolios. In fact, this means reducing the risk profile of the stock portfolio.

The above examples of admissible and effective sets are based on statistical information on the market value of shares in the FMTS and UM markets. Such information is concise and is provided to describe the computational capabilities of the stock portfolio diversification algorithm.

\section{CONCLUSION}

In this study, new mathematical statements of optimization of stock portfolio structure are presented and methods of their solution are developed. Mathematical problems formulated on the basis of models of the dynamics of market value of one share and portfolio of shares. That gives an opportunity to solve the problem of optimal diversification of the portfolio of investments, taking into account of quantitative and qualitative market restrictions on the structure of the portfolio.

\section{REFERENCES}

1. Sharpe W. F. Investments / W.F. Sharpe, G.J. Alexander, J.V. Bailey. - Prentice Hall PTR, 1998. - 992 p.

2. Garashchenko F.G. Quality analysis of mathematical models of investment management / F.G. Garashchenko, V.R. Kulian, V.V. Rutitskaya // Cybernetics and Computer Engineering. - 2005. - No. 148. - P. 3-10.

3. Garashchenko F.G. Modelling and Analysis of Investment Trends / F.G. Garashchenko, V.R. Kulian, V.V. Rutitskaya // Journal of Automation and Information. — New York: Connecticut. — 2011. — Vol. 43, Issue 12. - P. 48-58.

4. Zaychenko Yuri. Direct and dual problem of investment portfolio optimization under uncertainty / Yu. Zaychenko, I. Sydoruk // International Journal "Information Technologies \& Knowledge". — 2014. — Vol. 8, No. 3. - P. 225-242.

5. Zaychenko Y.P. Analysis of the investment portfolio on the basis of forecasting stock prices / Zaychenko Y.P., Malyhah Esfandyyarfard // Visnyk NTUU "KPI". Informatics, operation and computer science. - 2007. - No. 47. - P. 168-179.

6. Zaychenko $Y$. Analysis of multicriteria problem of the portfolio optimization based on prediction of the assets / Zaychenko Y., Sydoruk I. // Visnyk NTUU "KPI". Informatics, operation and computer science. - 2015. - No. 62. - P. 79-88.

7. Orlovsky S.A. Problems of decision making at fuzzy initial information / S.A. Orlovsky. - M.: Nauka, 1981. - 208 p.

Received 13.02.2020

From the Editorial Board: the article corresponds completely to submitted manuscript. 